\title{
Effect of Surgery on the Long-Term Functional Outcome of Moyamoya Disease: A Meta-Analysis
}

\author{
Zhong YAO, Chao YOU \\ Sichuan University, West China Hospital, Department of Neurosurgery, Chengdu, China \\ Corresponding author: Chao YOU youchaohx@aliyun.com
}

\section{ABSTRACT}

AIM: To summarize and calculate the accurate rate of good outcome and the prognosis, using a uniform definition of good outcome, evaluating the long-term effect of surgery.

MATERIAL and METHODS: Through searching the relevant databases, we included eligible studies measuring outcomes with the modified Rankin Scale (mRS), Glasgow Outcome Scale (GOS) and Kim's category. Single group rates were transformed and synthesized to yield the mean weighted probability. Sensitivity analyses were conducted to check the robustness of overall effect. Subgroup analyses were stratified by study type, population, age phase, presentation, surgical procedure and outcome measurement.

RESULTS: The overall estimated rate of good outcome was 0.87 ( $95 \% \mathrm{Cl}, 0.840 .90)$, with substantial heterogeneity. In the subgroup analyses, the outcome measured with GOS led to the highest rate of $91 \%$, while Kim's category had the lowest rate of $82 \%$. The combined bypass surgery group resulted in a higher good outcome rate of $0.92(95 \% \mathrm{Cl}, 0.890 .96)$ than the indirect bypass group of $0.83 \%(95 \% \mathrm{Cl}, 0.780 .88)$.

CONCLUSION: Moyamoya disease after surgical treatment could achieve a good outcome rate of $87 \%$. Kim's category reflected the functional outcome better, resulting in a good outcome rate of $82 \%$. The combined bypass surgery led to better outcomes than indirect bypass.

KEYWORDS: Long-term outcome, Meta-analysis, Moyamoya disease, Surgical treatment

\section{INTRODUCTION}

$\mathrm{M}$ oyamoya disease (MMD) is a cerebrovascular condition, characterized by progressive occlusion of bilateral internal carotid artery terminals and the compensatory formation of abnormal net-like vessels at the base of the brain (32). The incidence of MMD is about 0.35 per 100,000 population worldwide and increases to 3 per 100,000 in Asian population (31). Clinical presentations are mainly divided into two categories: ischemic symptoms (i.e., infarct, seizure and headache) and hemorrhagic symptoms. The pathogenesis of MMD remains unclear and etiological treatment is not yet established. Surgical treatment, including direct bypass, indirect bypass and combined bypass, has become the mainstay for MMD.
Studies have found revascularization surgery to contribute to promote collateral vessel formation and augment cerebral blood flow (17). However, some researchers found no correlation between the change of cerebral blood flow and the functional outcome $(3,38)$, making it questionable whether radiological manifestation should be used as a surrogate of the outcome or not. While most studies paid attention to the angiographic response and recurrent stroke rate, the long-term functional outcome was less focused. The various definitions of good outcome in different studies weakened the comparability between studies $(13,29)$. Besides, the conduction of compared trials was ethically difficult due to the confirmed benefit of surgery, especially in the ischemic MMD population $(7,22)$. 
The prognosis and exact rate of good functional outcome of MMD after operations were controversial. Hence, we conducted a meta-analysis to clarify the probability of good outcome under the uniform outcome measurement tools, in order to shed light on the effect of surgery on MMD outcomes.

\section{- MATERIAL and METHODS}

\section{Search Strategy}

We systematically searched databases through Pubmed, Ovid and Web of Science from their starting data up to July 2017. The detailed search strategy was shown in the Table I. The reference lists of extracted articles were also manually searched. Only English language articles were searched without time limitation.

\section{Study Selection}

We included studies meeting the following criteria:

[1] Observational studies describing patients with the diagnosis of MMD according to the diagnosis and treatment guideline (31)

[2] Patients that underwent revascularization surgery either by direct bypass, indirect bypass or combined bypass

[3] With an exact definition of good functional outcome

[4] Recording the number of patients with good outcome or the rate

[5] With follow-up time of at least 6 months.
Studies were excluded for the following reasons:

[1] Described quasi-Moyamoya or Moyamoya syndrome

[2] Sample size smaller than 10 patients.

Two researchers (Z.Y. and C.Y.) independently screened and selected eligible studies, and the discrepancy was solved by discussion and consensus.

\section{Uniform Definition of the Good Outcome}

In this meta-analysis, three common clinical outcome measurement tools of the modified Rankin Scale (mRS), Glasgow Outcome Scale (GOS) and Moyamoya Disease outcome category proposed by Kim et al.(18), were used as uniform standard measurement. Kim et al. classified the outcomes into four categories: Excellent, the preoperative symptoms had totally disappeared without fixed neurological deficits; good, the symptoms had totally disappeared, but neurological deficits remained; fair, the symptoms persisted, albeit less frequently; poor, where the symptoms remained unchanged or had worsened. Of these, categories of excellent and good were considered as favorable outcomes (18). According to usual practice, we defined good outcome as $m R S \leq 2, G O S>3$ and favorable outcomes in Kim's category.

\section{Quality Assessment}

All eligible studies underwent quality assessment with the methodological index for non-randomized studies (MINORS) (33). Every included study was scored 0 to 2 in each of eight items for non-comparative studies.

Table I: Detailed Search Strategy

\section{Database Search Strategy}

1. (surgery or revascularization or bypass or temporal muscle sticking or encephalo-myo-synangiosis or encephalo-duro-arterio-synangiosis or encephalo-duro-arterio-myo-synangiosis or encephalo-myo-arteriosynangiosis or encephalo-duro-arterio-galeo-synangiosis or superficial temporal artery-middle cerebral artery

Ovid anastomosis).tw.

2. (outcome or prognosis or mRS or modified rankin scale or GOS or Glasgow outcome scale).tw.

3. moyamoya.tw.

4. 1 and 2 and 3

$(((()(()((()($ surgery[Text Word]) OR revascularization[Text Word]) OR bypass[Text Word]) OR temporal muscle sticking[Text Word]) OR encephalo-myo-synangiosis[Text Word]) OR encephalo-duro-arterio-synangiosis[Text Word]) OR encephalo-duro-arterio-myo-synangiosis[Text Word]) OR encephalo-myo-arterio-synangiosis[Text Pubmed Word]) OR encephalo-duro-arterio-galeo-synangiosis[Text Word]) OR superficial temporal artery-middle cerebral artery anastomosis[Text Word])) AND moyamoya[Text Word]) AND ((((()outcome[Text Word]) OR prognosis[Text Word]) OR mRS[Text Word]) OR modified rankin scale[Text Word]) OR GOS[Text Word]) OR glasgow outcome scale[Text Word]))

\#4 \#3 AND \#2 AND \#1

\#3 TOPIC: (outcome) OR TOPIC: (prognosis) OR TOPIC: (mRS) OR TOPIC: (modified rankin scale) OR TOPIC: (GOS) OR TOPIC: (glasgow outcome scale)

Web of \#2 TOPIC: (moyamoya)

Science

\#1 TOPIC: (surgery) OR TOPIC: (revascularization) OR TOPIC: (bypass) OR TOPIC: (temporal muscle sticking) OR TOPIC: (encephalo-myo-synangiosis) OR TOPIC: (encephalo-duro-arterio-synangiosis) OR TOPIC: (encephalo-duro-arterio-myo-synangiosis) OR TOPIC: (encephalo-myo-arterio-synangiosis) OR TOPIC: (encephalo-duro-arterio-galeo-synangiosis) OR TOPIC: (superficial temporal artery-middle cerebral artery anastomosis) 


\section{Data Extraction}

Data extraction forms were used to collect relevant information including author names, years of publication, study types, study duration, countries, outcome measurement, follow-up time, sample sizes and rates of long-term good outcome. When the population in different studies totally or partially overlapped, we included the study with bigger sample size or more comprehensive data. We also tried to contact the authors of primary articles for intact data.

\section{Statistic Analyses}

Single group rates of good outcome were transformed with the variance-stabilizing Freeman-Tukey arcsine method. Transformed rates and standard errors were re-transformed back to probabilities in the random-effects model metaanalysis, yielding mean weighted probability (MWP) and 95\% confidence interval $(\mathrm{Cl})$. Heterogeneity was measured with Cochran's $Q$ and $I^{2}$ tests and the value of $I^{2}$ greater than $50 \%$ indicated pronounced heterogeneity. Begg's and Egger's tests were applied to calculate publication bias and a $p<0.05$ showed significant bias. Sensitivity analyses were conducted by omitting one study each time and synthesizing the estimates of reminding studies, so as to check the robustness of the overall effect. Subgroup analyses were stratified by study type, population, age phase, presentation, surgical procedure and outcome measurement. In the subgroup analyses of clinical presentations, characteristics of greater than $90 \%$ patients represented the trait of the whole population (i.e. if more than $90 \%$ patients exhibited ischemic symptom, we considered this whole population as the ischemic population). The contribution of variables to the heterogeneity was evaluated in the univariate regression analyses and a $p<0.05$ indicated significance. The summarized analyses of good outcomes were on the patient basis. All the statistic tests were implemented with Stata 14.0 software (Stata Cooperation USA).

\section{RESULTS}

\section{Literature Search}

After removing duplicates, a total of 1928 articles were screened, of which 64 records entered the full-text reading assessment for eligibility. Then 44 citations were excluded for certain reasons: review articles, without pertinent data, non-standard or self-defined outcome measurement, and overlapped population in different studies $(5,6,21,22,27,34)$. Finally, 20 observational studies were included in the data synthesis. The literature search process was outlined in Figure 1.

\section{Main Characteristics of Included Studies}

Totally we included 2666 MMD patients with a female predominance, most of which were from East Asian countries.

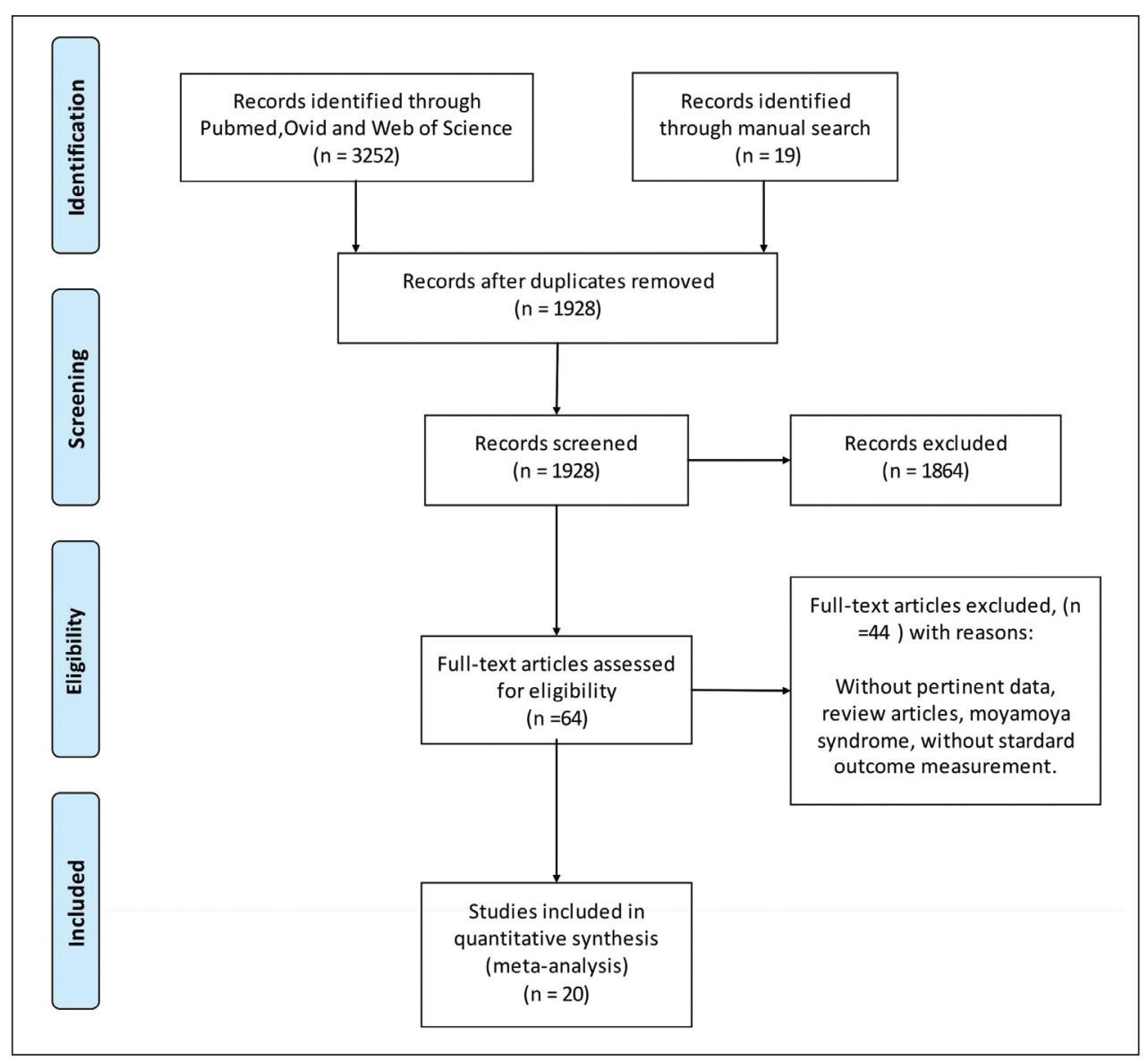

Figure 1: Flow diagram for literature search. 
Of all, 16 included studies were published after the date of 2012, when the diagnosis and treatment guideline was reported (31). The main surgery procedures are superficial temporal artery-middle cerebral artery bypass, encephaloduroarteriosynangiosis and modified operation procedures based on the encephaloduroarteriosynangiosis. The mean follow-up time varied ranging from 6 to 171 months. Most included studies were of high or moderate quality, with a mean score of 12 in MINORS. The detailed characteristics were depicted in the Table II.

Table II: Main Characteristics of Included Studies

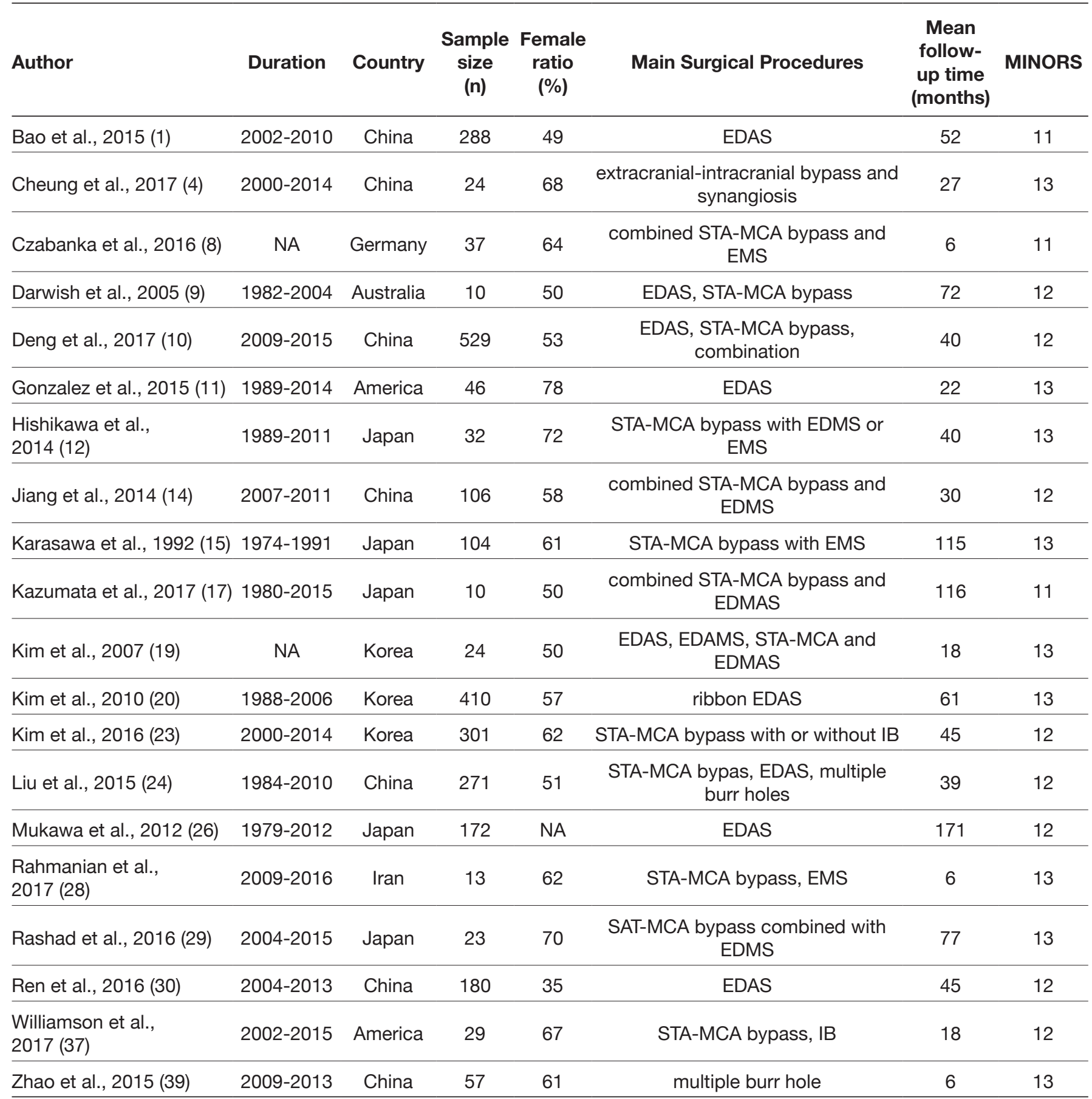

EDAS: encephaloduroarteriosynangiosis; EDMAS: encephaloduroarteriomyosynangiosis; EDMS: encephaloduromyosynangiosis; EMS: encephalomyosynangiosis; IB: indirect bypass; MINORS: methodological index for non-randomized studies; NA: not available; STA-MCA: superficial temporal artery-middle cerebral artery. 


\section{Meta-analyses}

The overall MWP was 0.87 (95\% Cl, 0.840 .90$)$ for all included studies and the heterogeneity was obvious between studies $\left(I^{2}=82.2 \%, p<0.0001\right.$, Figure 2$)$. In the sensitivity analyses by excluding any single study each time, overall MWP did not change significantly (Figure 3). Publication bias was not substantial in Egger's test $(\mathrm{p}=0.09)$, though the funnel plot was slightly asymmetric (Figure 4).

Stratified by outcome measurement, the MWP was 0.82 $(95 \% \mathrm{Cl}, 0.800 .85)$ when the outcome measured by Kim's category, and MWP increased to $0.91(95 \% \mathrm{Cl}, 0.820 .99)$ when measured by GOS. The heterogeneity was 0 and $71.7 \%$ in Kim's category and GOS subgroups respectively (Figure 2). The most commonly used outcome measurement tool was mRS (12 studies), resulting in the MWP of $0.89(95 \% \mathrm{Cl}$, 0.85 0.93). In addition, non-significant differences of MWP existed between prospective and retrospective studies, Asian and non-Asian populations, children and adults, ischemic and hemorrhagic presentations. 273 patients in six studies underwent combined bypass surgery and 1377 patients in eight studies experienced indirect bypass, but no studies documenting solo direct bypass were included. The higher MWP was shown in the combined bypass surgery group

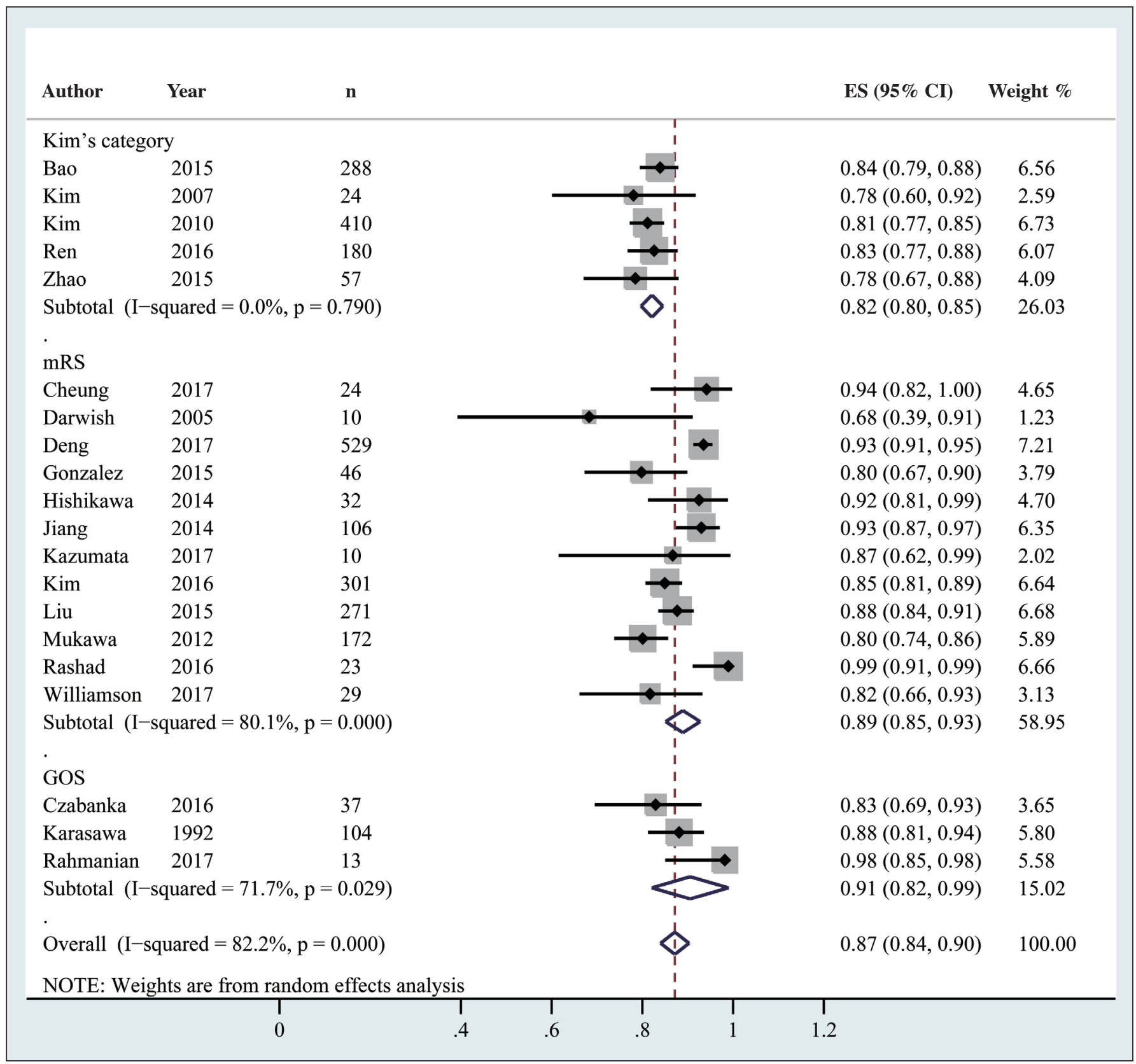

Figure 2: Forest plot of studies stratified by outcome measurement, with single group rates, synthesized mean weighted probability and $95 \%$ confidence intervals. 
$(0.92[95 \% \mathrm{Cl}, 0.890 .96])$ than in the indirect bypass group $(0.83[95 \% \mathrm{Cl}, 0.780 .88])$, without intersections between these two Cls (Table III). Substantial heterogeneity existed within indirect bypass studies $\left(I^{2}=83 \%, p<0.0001\right)$, but not in combined bypass studies $\left(I^{2}=0, p=0.61\right)$. In the regression analyses, variables of study type, population and outcome measurement exhibited no significant relationship with the overall MWP, contributing little to the heterogeneity.

\section{DISCUSSION}

The overall estimated rate of good outcome was $87 \%$ under the uniform outcome measurement. In the subgroups, measurement by GOS showed the highest good outcome rate, while measurement by Kim's category indicated the lowest rate. This was in line with previous study which evaluated the same population with two outcome measurement tools achieving different results (30). In spite of heterogeneity, the combined bypass surgery brought about a significant higher good outcome rate than the indirect bypass did.
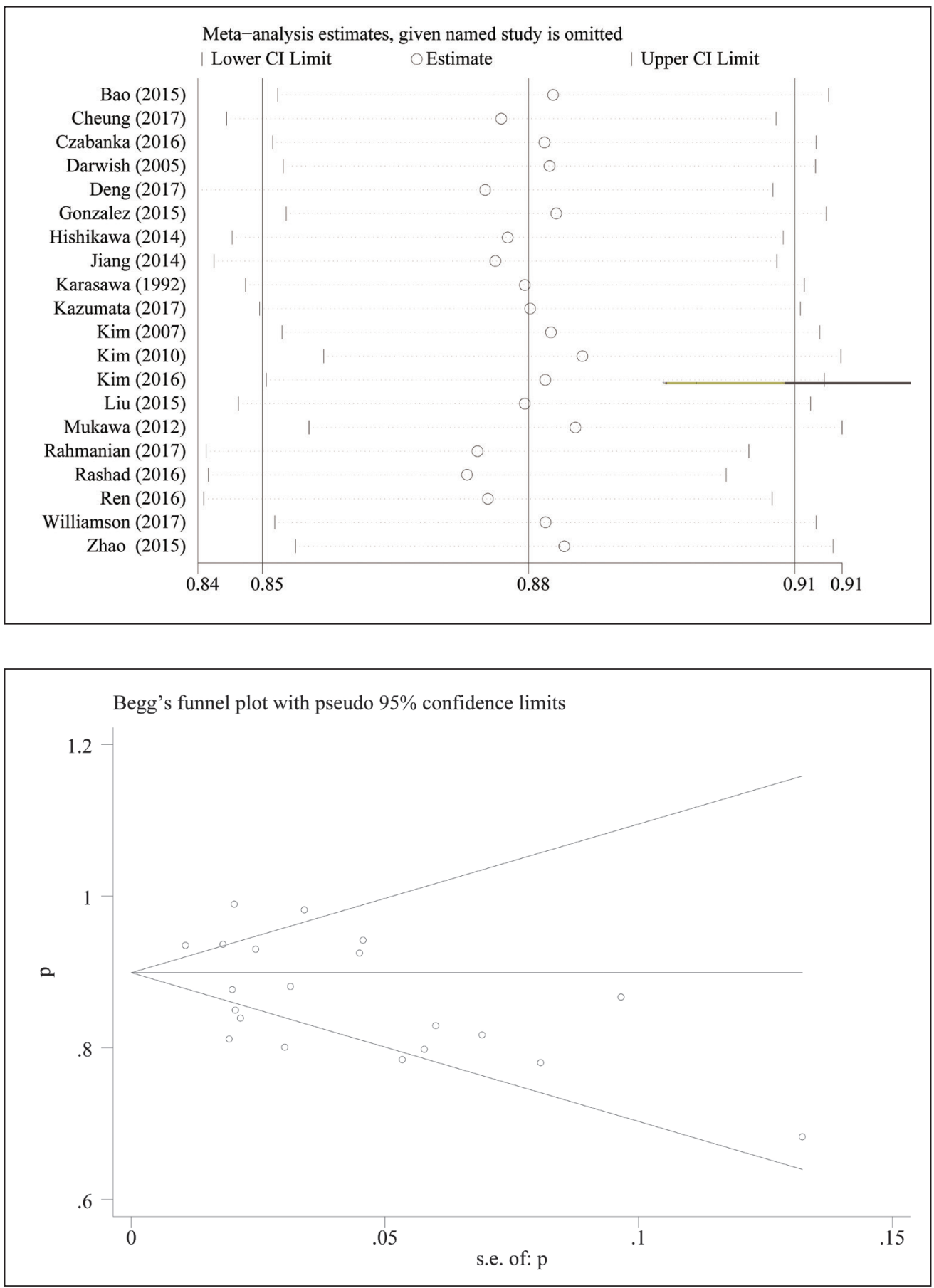

Figure 3: Sensitivity analyses by excluding single one study each time.
Figure 4: Begg's funnel plot for publication bias. 
The regression analyses found no variables contributing significantly to the heterogeneity, which might be explained by the within studies variance as patients' different reactions to surgery and variation of operations. After all, the heterogeneity was not uncommon in the meta-analysis of single group rate due to the one-arm trait (2).

MMD was complicated by the recurrent postoperative stroke, and thus the long-term functional outcome became the important prognosis index. Surgical treatment aimed to augment blood flow to the affected cerebral hemispheres, increase perfusion and improve clinical outcomes $(1,4)$. The collateral circulation took approximate six months to develop well after the operations (25), for which we adopted six months as the shortest follow-up time in the including criteria. The previous review summarized and calculated the mean estimated proportion of the postoperative stroke in MMD was 5.4\% (16). Further studies pointed out bypass surgery effectively decreased the occurrence of stroke (24). To what extent the operations were able to improve the long-term functional outcome was under debate. Researchers noted the evaluation of surgical effects on the outcomes of MMD patients was difficult, for surgery was a palliative form in a progressive disease with poor natural history (35).
Sun et al. figured out the rates of favorable outcome were $80 \%$, $78 \%$ and $81 \%$ in direct, indirect and combined bypass groups respectively (36), but the overall estimates were of obvious heterogeneity due to the various definitions of favorable outcomes. Here, we calculated the mean rate of good outcome with uniform measurement tools and definitions, resulting in a higher rate of $87 \%$. In the review, we excluded studies using self-defined good outcome measurement, which might underestimate the good functional outcome rate. Moreover, we found a higher good outcome rate existed in studies using GOS than those using Kim's category. GOS was used to reflect the ability for daily activity, giving little attention to the recurrent symptoms in MMD. Kim's category was exclusively to measure the outcome of MMD, taking both the recurrent symptoms and the residual functional inability into consideration. The estimated rate of good outcome measured by Kim's category was closer to the previous reported rate. We deemed Kim's category could better reflect MMD patient's prognosis. Besides, combined bypass surgery led to a higher good outcome rate than indirect bypass surgery, in accordance with previous study (10). It was well acknowledged that direct and combined bypass could generate better collateral vessel formation. The main reason impeding the application of

Table III: Subgroup Analyses by Study Type, Population, Age Phase, Presentation, Surgical Procedure and Outcome Measurement

\begin{tabular}{|c|c|c|c|c|c|c|}
\hline & $\begin{array}{l}\text { Number of } \\
\text { studies }\end{array}$ & $\begin{array}{c}\text { Number of } \\
\text { patients }\end{array}$ & $I^{2}(\%)$ & $p$ for heterogeneity & MWP (95\% Cl) & Weight (\%) \\
\hline \multicolumn{7}{|l|}{ Study type } \\
\hline Retrospective & 18 & 2031 & 78 & $<0.0001$ & $0.86(0.83,0.90)$ & 86 \\
\hline Prospective & 2 & 635 & 0 & 0.85 & $0.93(0.91,0.95)$ & 14 \\
\hline \multicolumn{7}{|l|}{ Population } \\
\hline Asian & 16 & 2544 & 84 & $<0.0001$ & $0.88(0.85,0.91)$ & 88 \\
\hline Non-Asian & 4 & 122 & 0 & 0.79 & $0.81(0.74,0.87)$ & 12 \\
\hline \multicolumn{7}{|l|}{ Age phase } \\
\hline Children & 7 & 1031 & 89 & $<0.0001$ & $0.85(0.78,0.92)$ & 51 \\
\hline Adults & 7 & 731 & 56 & 0.04 & $0.86(0.82,0.90)$ & 49 \\
\hline \multicolumn{7}{|l|}{ Presentation } \\
\hline Ischemic & 15 & 1782 & 83 & $<0.0001$ & $0.87(0.83,0.91)$ & 84 \\
\hline Hemorrhagic & 2 & 248 & 0 & 0.61 & $0.92(0.89,0.95)$ & 16 \\
\hline \multicolumn{7}{|l|}{ Surgical procedure } \\
\hline Indirect bypass & 8 & 1377 & 83 & $<0.0001$ & $0.83(0.78,0.88)$ & 61 \\
\hline Combined bypass & 6 & 273 & 0 & 0.61 & $0.92(0.89,0.96)$ & 39 \\
\hline \multicolumn{7}{|l|}{ Outcome measurement } \\
\hline Kim's category & 5 & 959 & 0 & 0.79 & $0.82(0.80,0.85)$ & 26 \\
\hline $\mathrm{mRS}$ & 12 & 1553 & 80 & $<0.0001$ & $0.89(0.85,0.93)$ & 59 \\
\hline GOS & 3 & 154 & 71 & 0.03 & $0.91(0.82,0.99)$ & 15 \\
\hline
\end{tabular}

Cl: Confidence interval; GOS: Glasgow Outcome Scale; mRS: Modified Rankin Scale; MWP: Mean Weighted Probability 
combined bypass was the lack of eligible arteries, especially in children. Moreover, postoperative hyperperfusion syndrome should also be noticed.

There were several limitations in this meta-analysis. Firstly, patients' conditions on admission were different among the included studies. Besides, substantial heterogeneity could not be fully explained and further studies were warranted.

\section{CONCLUSION}

The overall estimated rate of good outcome was $87 \%$ in MMD after operations. Kim's category reflected the functional outcome accurately, resulting in a good outcome rate of $82 \%$. Combined bypass surgery brought about a higher good outcome rate than the indirect bypass did.

\section{REFERENCES}

1. Bao XY, Duan L, Yang WZ, Li DS, Sun WJ, Zhang ZS, Zong $\mathrm{R}$, Han C: Clinical features, surgical treatment, and long-term outcome in pediatric patients with moyamoya disease in China. Cerebrovasc Dis 39:75-81, 2015

2. Beijer $U$, Wolf $A$, Fazel S: Prevalence of tuberculosis, hepatitis $C$ virus, and HIV in homeless people: A systematic review and meta-analysis. Lancet Infect Dis 12:859-870, 2012

3. Caldarelli M, Di Rocco C, Gaglini P: Surgical treatment of moyamoya disease in pediatric age. J Neurosurg Sci 45:8391, 2001

4. Cheung AH, Lam AK, Ho WW, Tsang CP, Tsang AC, Lee R, Lui WM, Leung GK: Surgical outcome for Moyamoya Disease: Clinical and perfusion computed tomography correlation. World Neurosurg 98:81-88, 2017

5. Cho A, Chae JH, Kim HM, Lim BC, Hwang H, Hwang YS, Phi JH, Kim SK, Wang KC, Cho BK, Kim KJ: Electroencephalography in pediatric moyamoya disease: Reappraisal of clinical value. Childs Nerv Syst 30:449-459, 2014

6. Cho HH, Cheon JE, Kim SK, Choi YH, Kim IO, Kim WS, Lee SM, You SK, Shin SM: Quantitative assessment of neovascularization after indirect bypass surgery: Colorcoded digital subtraction angiography in pediatric Moyamoya Disease. AJNR Am J Neuroradiol 37:932-938, 2016

7. Cho WS, Kim JE, Kim CH, Ban SP, Kang HS, Son YJ, Bang JS, Sohn CH, Paeng JC, Oh CW: Long-term outcomes after combined revascularization surgery in adult moyamoya disease. Stroke 45:3025-3031, 2014

8. Czabanka M, Boschi A, Acker G, Pena-Tapia P, Schubert GA, Schmiedek P, Vajkoczy P: Grading of moyamoya disease allows stratification for postoperative ischemia in bilateral revascularization surgery. Acta Neurochir (Wien) 158:18951900, 2016

9. Darwish B, Besser M: Long term outcome in children with Moyamoya disease: Experience with 16 patients. J Clin Neurosci 12:873-877, 2005

10. Deng X, Gao F, Zhang D, Zhang Y, Wang R, Wang S, Cao Y, Zhao Y, Pan Y, Ye X, Liu X, Zhang Q, Wang J, Yang Z, Zhao M, Zhao J: Effects of different surgical modalities on the clinical outcome of patients with moyamoya disease: A prospective cohort study. J Neurosurg 2017 (Epub Ahead of Print)
11. Gonzalez NR, Dusick JR, Connolly M, Bounni F, Martin NA, Van de Wiele B, Liebeskind DS, Saver JL: Encephaloduroarteriosynangiosis for adult intracranial arterial steno-occlusive disease: Long-term single-center experience with 107 operations. J Neurosurg 123:654-661, 2015

12. Hishikawa T, Tokunaga K, Sugiu K, Date I: Long-term outcomes in adult patients with ischemic-type moyamoya disease involving posterior circulation. Acta Neurochir (Wien) 156:1745-1751, 2014

13. Imaizumi T, Hayashi K, Saito K, Osawa M, Fukuyama Y: Longterm outcomes of pediatric moyamoya disease monitored to adulthood. Pediatr Neurol 18:321-325, 1998

14. Jiang H, Ni W, Xu B, Lei Y, Tian Y, Xu F, Gu Y, Mao Y: Outcome in adult patients with hemorrhagic moyamoya disease after combined extracranial-intracranial bypass. J Neurosurg 121:1048-1055, 2014

15. Karasawa J, Touho H, Ohnishi H, Miyamoto S, Kikuchi H: Long-term follow-up study after extracranial-intracranial bypass surgery for anterior circulation ischemia in childhood moyamoya disease. J Neurosurg 77:84-89, 1992

16. Kazumata K, Ito M, Tokairin K, Ito Y, Houkin K, Nakayama $\mathrm{N}$, Kuroda S, Ishikawa T, Kamiyama H: The frequency of postoperative stroke in moyamoya disease following combined revascularization: A single-university series and systematic review. J Neurosurg 121:432-440, 2014

17. Kazumata K, Kamiyama H, Saito H, Maruichi K, Ito M, Uchino $\mathrm{H}$, Nakayama N, Kuroda S, Houkin K: Direct anastomosis using occipital artery for additional revascularization in Moyamoya Disease after combined superficial temporal artery-middle cerebral artery and indirect bypass. Oper Neurosurg 13:213222, 2017

18. Kim CY, Wang KC, Kim SK, Chung YN, Kim HS, Cho BK: Encephaloduroarteriosynangiosis with bifrontal encephalogaleo (periosteal) synangiosis in the pediatric moyamoya disease: The surgical technique and its outcomes. Childs Nerv Syst 19:316-324, 2003

19. Kim DS, Kang SG, Yoo DS, Huh PW, Cho KS, Park CK: Surgical results in pediatric moyamoya disease: Angiographic revascularization and the clinical results. Clin Neurol Neurosurg 109:125-131, 2007

20. Kim SK, Cho BK, Phi JH, Lee JY, Chae JH, Kim KJ, Hwang YS, Kim IO, Lee DS, Lee J, Wang KC: Pediatric moyamoya disease: An analysis of 410 consecutive cases. Ann Neurol 68:92-101, 2010

21. Kim SK, Seol HJ, Cho BK, Hwang YS, Lee DS, Wang KC: Moyamoya disease among young patients: Its aggressive clinical course and the role of active surgical treatment. Neurosurgery 54:840-845, 2004

22. Kim SK, Wang KC, Kim IO, Lee DS, Cho BK: Combined encephaloduroarteriosynangiosis and bifrontal encephalogaleo (periosteal) synangiosis in pediatric moyamoya disease. Neurosurgery 50:88-96, 2002

23. Kim T, Oh CW, Kwon OK, Hwang G, Kim JE, Kang HS, Cho WS, Bang JS: Stroke prevention by direct revascularization for patients with adult-onset moyamoya disease presenting with ischemia. J Neurosurg 124:1788-1793, 2016 
24. Liu XJ, Zhang D, Wang S, Zhao YL, Teo M, Wang R, Cao Y, Ye $X$, Kang S, Zhao JZ: Clinical features and long-term outcomes of moyamoya disease: A single-center experience with 528 cases in China. J Neurosurg 122:392-399, 2015

25. Matsushima Y, Inaba Y: Moyamoya disease in children and its surgical treatment. Introduction of a new surgical procedure and its follow-up angiograms. Childs Brain 11:155-170, 1984

26. Mukawa M, Nariai T, Matsushima Y, Tanaka Y, Inaji M, Maehara T, Aoyagi M, Ohno K: Long-term follow-up of surgically treated juvenile patients with Moyamoya disease. J Neurosurg Pediatr 10:451-456, 2012

27. Park JH, Yang SY, Chung YN, Kim JE, Kim SK, Han DH, Cho BK: Modified encephaloduroarteriosynangiosis with bifrontal encephalogaleoperiosteal synangiosis for the treatment of pediatric moyamoya disease. Technical note. J Neurosurg 106:237-242, 2007

28. Rahmanian A, Masoudi MS, Ghaffarpasand F, Ashraf MH, Alibai E: Outcome of microsurgical revascularization in patients with Moyamoya Disease; First report from Iranian population. Turk Neurosurg 2017 (Epub ahead of print)

29. Rashad S, Fujimura M, Niizuma K, Endo H, Tominaga T: Long-term follow-up of pediatric moyamoya disease treated by combined direct-indirect revascularization surgery: Single institute experience with surgical and perioperative management. Neurosurg Rev 39:615-623, 2016

30. Ren B, Zhang ZS, Liu WW, Bao XY, Li DS, Han C, Xian P, Zhao F, Wang $\mathrm{H}$, Wang $\mathrm{H}$, Duan L: Surgical outcomes following encephaloduroarteriosynangiosis in adult moyamoya disease associated with Type 2 diabetes. J Neurosurg 125:308-314, 2016

31. Research Committee on the P, Treatment of Spontaneous Occlusion of the Circle of W, Health Labour Sciences Research Grant for Research on Measures for Infractable D: Guidelines for diagnosis and treatment of moyamoya disease (spontaneous occlusion of the circle of Willis). Neurol Med Chir (Tokyo) 52:245-266, 2012
32. Scott RM, Smith ER: Moyamoya disease and moyamoya syndrome. N Engl J Med 360:1226-1237, 2009

33. Slim K, Nini E, Forestier D, Kwiatkowski F, Panis Y, Chipponi $\mathrm{J}$ : Methodological index for non-randomized studies (minors): Development and validation of a new instrument. ANZ J Surg 73:712-716, 2003

34. So Y, Lee HY, Kim SK, Lee JS, Wang KC, Cho BK, Kang E, Lee DS: Prediction of the clinical outcome of pediatric moyamoya disease with postoperative basal/acetazolamide stress brain perfusion SPECT after revascularization surgery. Stroke 36:1485-1489, 2005

35. Starke RM, Komotar RJ, Hickman ZL, Paz YE, Pugliese AG, Otten ML, Garrett MC, Elkind MS, Marshall RS, Festa JR, Meyers PM, Connolly ES Jr: Clinical features, surgical treatment, and long-term outcome in adult patients with moyamoya disease. Clinical article. J Neurosurg 111:936-942, 2009

36. Sun H, Wilson C, Ozpinar A, Safavi-Abbasi S, Zhao Y, Nakaji P, Wanebo JE, Spetzler RF: Perioperative complications and long-term outcomes after bypasses in adults with Moyamoya Disease: A systematic review and meta-analysis. World Neurosurg 92:179-188, 2016

37. Williamson RW, Abla AA, Zabramski JM, Nakaji P, Spetzler RF, Wanebo JE: Revascularization of Moyamoya angiopathy in older adults. World Neurosurg 99:37-40, 2017

38. Yun TJ, Cheon JE, Na DG, Kim WS, Kim IO, Chang KH, Yeon KM, Song IC, Wang KC: Childhood Moyamoya Disease: Quantitative evaluation of perfusion MR imaging--correlation with clinical outcome after revascularization surgery. Radiology 251:216-223, 2009

39. Zhao X, Wang C, Ji Y, Han C, Wang M: Therapeutic effect of multiple burr hole operation combined with dural inversion and periosteal synangiosis for Moyamoya Disease. Br J Neurosurg 29:811-817, 2015 